\title{
Pierre Franco (1505-1578) : andrologue avant la lettre par la création de la cure chirurgicale des hernies sans ablation du testicule
}

\author{
Georges ANDROUTSOS \\ Histoire de la Médecine, Facultè de Médecine, Université d'loannina, Grèce
}

Mots clés : Pierre Franco, chirurgien des hernies, taille hypogastrique, andrologie «Endurer pour durer» Pierre Franco

\section{INTRODUCTION}

$\mathrm{Au} 16^{\mathrm{e}}$ siècle allait surgir une forte personnalité, un pauvre inciseur de pierres et opérateur herniaire, Pierre Franco, qui créa la procédure de guérison des hernies sans oter les parties génitales : progrès capital, qui inaugura la suppression de la castration, cause principale de stérilité et d'impuissance.

\section{GENERALITES}

Pierre Franco naquit entre 1500 et 1505 dans le petit village de Turriers (Haute-Provence). II fut en apprentissage chez un opérateur herniaire, et ne reçut aucune instruction théorique. II resta un opérateur, c'est-à-dire que bien qu'il fût appelé maître, il était au-dessous des barbiers-chirurgiens (titre que portait Paré). Vers 1541, il quitta la France pour se réfugier en Suisse où il vécut à Berne et à
Lausanne. Franco fut, selon les termes de l'autorisation qui l'admit à exercer à Lausanne, qualifié comme tailleur de vessies, de hernies et de cataractes [1]. Ayant probablement adopté la réforme de Calvin, il fuyait le début des persécutions religieuses. En 1559 il revient en France, séjourne à Lyon et à Orange, profitant d'une trêve courte. Car, en 1562 , Orange est mise à sac par les troupes catholiques. Et c'est un nouveau refuge à Lausanne, où il meurt vers 1578 [7].

Franco a publié deux ouvrages, en 1556 et en 1561 . Celui de 1556 intitulé Petit Traité Contenant Une Des Parties Principales De Chirurgie, Laquelle Les Chirurgiens Hernières Exercent est assez succinct et ne traite guère que des matières dans lesquelles Franco s'est, pour ainsi dire, spécialisé : les hernies, la pierre, la cataracte [5]. L'ouvrage de 1561 s'intitule Traité Des Hernies (Figure 1) est beaucoup plus développé [6]. Cet ouvrage contient 544 pages et a constitué la première étude complète sur l'hernie et ses thérapies opératoires et non-invasives. De plusieurs autres matières chirurgicales importantes, telles que des maladies génito-urinaires, des difficultés ophtalmologiques, des lésions traumatiques, et des fractures et des dislocations, y compris des amputations, ont été également discutées [4].

\section{LE TRAITEMENT DES HERNIES AVANT FRANCO}

Quand Franco commenca à exercer, on traitait souvent encore les hernies par « voye de médecine »; Franco conseillait, lui aussi, quand les hernies n'étaient pas trop invétérées ou encore incomplètes d'essayer cette " curation par médecines tant prises en dedans qu'appliquées

\section{Correspondance :}

Dr Georges ANDROUTSOS, 1 rue Ipeirou, 10433, Athènes, Grèce - Fax +30-210-8235710 - Email paris48@otenet.gr 


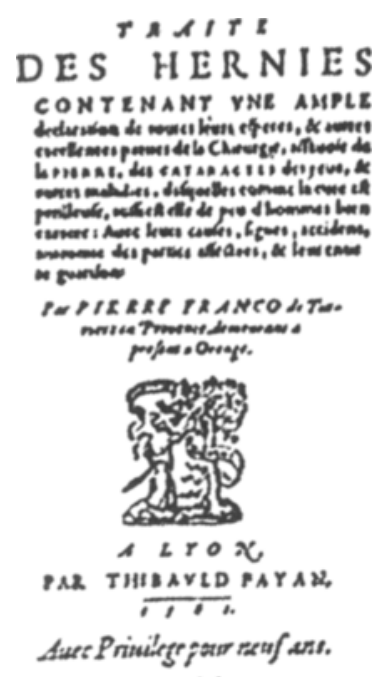

(A)

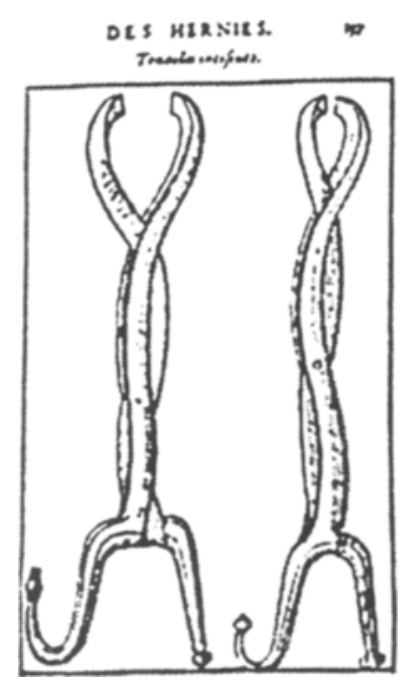

Figure 1 : Page de front et instruments chirurgicaux du Traité des hernies (1561) [6].

au-dehors avec emplâtres trempées dans des astringents "; mais il s'élèva contre cette méthode si l'on échouait ou quand la hernie était complète ou invétérée et prônait alors la cure par chirurgie [8].

Jusqu'à l'époque de Franco, la hernie dont les formes cliniques étaient assez mal discernées, était traitée par la seule méthode chirurgicale dont il existait diverses variantes mais dont un temps était commun à presque toutes, l'ablation du testicule. II semble qu'un siècle avant Franco, un empirique espagnol en avait tenté la conservation. Certains opérateurs de hernies prétendaient le conserver ; en réalité, ils l'enlevaient et afin que l'entourage ne s'en aperçût pas, ils le jetaient sous la table et un chien dressé à cela en faisait son régal.

Aussi l'opération de hernie gardait-elle une réputation de gravité et la mutilation qui en résultait était-elle redoutée. L'introduction des bandages herniaires (brayers) ne fut adoptée vraiment que vers le $15^{\mathrm{e}}$ siècle. Elle fut par cer- tains considérée comme un progrès et cela amena une diminution des indications chirurgicales. Car le brayer métallique ou non fut considéré comme capable d'amener non seulement la contention, mais encore la guérison radicale de la hernie [3].

\section{FRANCO : CELEBRE HERNIOTOMISTE}

Pour suivre plus facilement les descriptions opératoires de Franco, il est utile de savoir ce qu'il écrit sur l'anatomie de la région inguinale et du scrotum et de se rendre compte de la façon dont il comprend la constitution et la disposition des enveloppes des bourses.

Franco ne connaissait que trois enveloppes des bourses : la peau, le dartos, l'érythroïde. II savait qu'en cas de hernie il s'y ajoute le péritoine, refoulé de l'abdomen par cette hernie (mais il ne semble pas avoir su qu'à l'état normal il existait autour du testicule une séreuse : la vaginale). Pour préciser : il semble bien qu'il faille comprendre qu'il appellait dartos ce que nous appelons encore aujourd'hui dartos et érythroïde la fibreuse recouverte de l'épanouissement en éventail du crémaster (qui, pour lui, ne descendrait pas dans le scrotum).

Franco, en écrivant aussi bien pour des opérateurs familiarisés avec les termes anatomiques que pour ceux qui n'en avaient aucune notion et qui employaient des désignations sans aucune précision, croyait devoir se mettre à leur portée en empruntant leur langage. Et c'est ainsi qu'il usait pour désigner les enveloppes rencontrées lors d'une opération de hernie du terme de didyme, qu'il savait pourtant signifier testicule [2].

Franco eut le mérite de comprendre l'importance du traitement chirurgical des hernies simples et étranglées, de la conservation du testicule et ainsi fit preuve d'un esprit didactique en étudiant les différentes variétés anatomopathologiques de cette affection. Sa classification anatomoclinique est la suivante : 1) hernie intestinale (entérocèle) ou scrotale ; 2) hernie inguinale (bubonocèle) ou ronde qui ne suit pas le didyme, c'est-à-dire le cordon spermatique (il a confondu là trois formes reconnues actuellement : la pointe de hernie inguinale indirecte, la hernie directe et la hernie crurale) ; 3) hernie zirbale ou épiploïque (en la palpant on a l'impression de toucher de la laine alors que la hernie intestinale est plus ferme, douloureuse et se réduit avec gargouillement). II y ajoute d'autres variétés auxquelles il donne le nom de hernies bien qu'elles n'en soient pas : hernie aqueuse, c'est-à-dire hydrocèle pour laquelle il décrit le signe de la transillumination avec une chandelle allumée pour savoir s'il n'y a pas d'intestin ou d'épiploon dedans ; hernie charneuse ou sarcocèle qui est une tumeur hors nature du testicule ; hernie variqueuse, c'est-à-dire le varicocèle qu'il traite par la section des veines entre deux ligatures [14].

Cette classification surprend au premier abord puisqu'elle est fondée tantôt sur le contenu, tantôt sur le siège ; elle se comprendra après lecture du texte de Franco. Pour distinguer ces hernies, et dans toute leur description, il se montre 
clinicien très avisé et enseigne avec précision et clairement une séméiologie très complète et très exacte [10].

Dans la préparation à l'intervention un détail mérite d'être noté : le patient étant purgé ou phlébotomisé, on ne doit pas l'opérer le lendemain, mais seulement le surlendemain « à cause que la médecine envoie quelques vapeurs et le corps est encore ému ». II y a peu de temps qu'on s'est insurgé à nouveau contre les purgations déshydratantes précédant de trop peu l'acte opératoire et favorisant le choc.

Après réduction de l'intestin dans le ventre, Franco attire alors le testicule nettement en dehors et le plus possible du didyme (c'est-à-dire du cordon et des enveloppes). Une tenaille spéciale le saisit, puis il est lié, avec toutes sortes de précautions, qu'il recommande pour éviter une hémorragie ou un lâchage des sutures, et enfin coupé.

L'incision se faisait jusqu'à Franco au niveau du pénil, c'està-dire dans la région inguinale (au niveau du collet) comme nous le faisons actuellement. Franco a imaginé de faire l'incision au niveau du scrotum et les raisons qu'il en donne sont parfaitement valables à son époque : il y a moins de danger d'hémorragie (on connaît, en effet, la riche vascularisation de l'aine), il y a moins de douleur puisque l'abord par le scrotum est plus direct et n'oblige à sectionner des nerfs, des muscles et de la graisse; de plus dans les suites puisqu'on laisse le scrotum largement ouvert on ne risque pas l'accumulation de sang ou l'apparition d'un phlegmon à son niveau. Ayant ainsi abordé le didyme ou sac on tire sur lui « en suffisance pas trop pour ne pas atteindre les chairs du pénil, assez pour ne pas laisser de sac amorce de récidive ". Prendre le sac avec les tenailles de hernie sans trop le serrer pour éviter contusion ou douleur, coudre le sac tout près de la tenaille au dessus d'elle; desserrer la tenaille au moment où on noue le fil et faire un deuxième nœud. Couper le didyme assez près du fil. Cette technique comporte l'ablation du testicule [9].

La suture du sac était fréquemment faite avec le point doré, c'est-à-dire un fil d'or qui donnait plus de garantie de solidité et aussi sans doute d'asepsie. Lorsque de l'épiploon devait être réséqué, Franco insiste sur la nécessité de le lier et le cautériser avant de le rentrer dans le ventre. Si c'est de l'intestin qui adhère au sac, il faut faire une ouverture assez grande mais prudente, introduire le doigt pour agrandir l'ouverture puis désagglutiner au doigt. Franco a étudié de près cette forme compliquée d'adhérences : il les attribue à une humidité visqueuse ou des excoriations causées par la réduction fréquente des intestins. II a remarqué que dans certains cas, par la faute des adhérences, la hernie ne peut se réduire mais l'orifice étant large il n'y a pas d'accidents graves. Il semble avoir été ainsi amené à mieux interpréter les accidents qui surviennent au niveau des hernies et considérés comme inflammation du scrotum avec gangrène. II donne une bonne description des signes généraux et locaux de la rétention dans le scrotum évoluant vers des troubles sphacéliques.

II semble bien que Franco aît été le premier à pratiquer la cure de la hernie étranglée, sûrement de la décrire. Voici sa déscription : après incision de la peau, introduire un bâton entre le sac et la chair du pénil et couper celle-ci. Tenter alors de réduire les intestins en pressant modérément et sans ouvrir le sac. Si cette manœuvre échoue, on ouvre le sac toujours sur le bâton jusqu'au trou du péritoine. "Ouvrir le péritoine sans rien craindre, prendre quelque linge suave et essayer de remettre les intestins peu à peu, commençant à ceux qui sont plus hauts vers le péritoine et le ventre ". C'est la kélotomie telle qu'on la pratique encore. L'avoir conçue et réalisée au début du $16^{e}$ siècle était une entreprise audacieuse et aurait dû assurer à son auteur une gloire définitive.

Franco connaissait les accidents qui adviennent à la hernie intestinale scrotale. II décrit bien les adhérences qu'on peut rencontrer de son contenu au sac et indique les mancuvres opératoires que doit exécuter le chirurgien pour les libérer sans danger. Mais il faut surtout mettre en valeur le rôle de Franco dans le traitement de " la rétention de la matière fécale dans le scrotum ", c'est-à-dire de ce que nous appelons la hernie étranglée.

Est-il premier à avoir opéré une hernie étranglée ? II semble qu'il y ait eu avant lui des tentatives de traitement opératoire de cette complication. Mais la description qu'il a donnée de cette opération est très vraisemblablement la première qu'on en connaisse. Et puis, il ne faut pas oublier qu'il opérait depuis environ trente ans quand il écrivit ces pages et que son expérience chirurgicale de cette technique était antérieure à la publication de son ouvrage.

II connaît bien entendu le pronostic fatal de cet accident abandonné à lui-même, qui commande l'intervention ; mais l'indication opératoire a pour lui des limites, limites justifiées du moins pour son époque où l'on ne songeait guère encore à la possibilité de faire une résection intestinale.

Si la partie, dit-il, n'a pas changé de couleur et est « en long ", si les moyens médicaux ont échoué, il faut intervenir ; mais si le scrotum a changé de couleur et est devenu noir, livide ou bleu et que la hernie soit plutôt " en rond qu'en long ", il ne faut pas entreprendre de la guérir car ces signes sont mauvais. On reconnaît là, en effet, la hernie avec gangrène intestinale, contre laquelle il se savait impuissant.

II décrit minutieusement deux techiniques opératoires. Dans l'une il opère en dehors du sac ; après une incision haute, il introduit un petit bâton rond en bout, plat d'un côté, entre le didyme et la chair du pénil qu'il coupe sur le côté plat du bâton et il essaie de réduire l'intestin.

Mais, s'il ne peut y parvenir, il faut, dit-il, couper sur l'ongle avec précaution le didyme (les enveloppes, péritoine compris) jusqu'à l'intestin et sectionner alors ce didyme sur le bâton introduit entre lui et l'intestin jusqu'au péritoine au point où cet intestin commence à descendre vers le scrotum " en faisant une bonne ouverture à ce péritoine " (c'est-àdire mener la section vers le haut jusqu'au péritoine abdominal libre).

Franco décrit, aussi, la hernie zirbale ou épiplocèle. Enfin, il décrit la hernie inguinale ou bubonocèle. Franco fut sans doute le premier à décrire une hernie crurale. La lecture de 
son intervention confime qu'il s'agit donc de la description d'une hernie crurale et de son opération. Mais pourquoi l'at-il appelée inguinale ? Et pourquoi, a-t-il classé les hernies, les deux premières d'après leur contenu, et cette dernière d'après son siège ?

On peut l'expliquer car il décrit précisément et incontestablement une variété nouvelle en ce qu'elle ne descend jamais comme les précédentes dans le scrotum où elles atteignent le gros volume permettant d'en distinguer aisément le contenu. Mais il ne pouvait pas lui donner le nom de crurale. En effet, on ne connaissait alors anatomiquement ni canal inguinal ni anneau crural. On ne savait donc pas que des hernies pouvaient sortir par deux orifices pariétaux différents et en tirer leur nom. Pour Franco, la désignation inguinale veut dire seulement située dans la région " des eines que les Latins appellent Inguina ", dit-il, et y restant, et il y ajoute bubonocèle parce que, peu volumineuse, elle ressemble à un bubon, affection qu'il connaisait déjà.

Aucune notion semblable de hernie située dans la région inguinale et y restant, mais n'étant pas le premier temps d'une future hernie scrotale, n'avait été faite avant lui [15].

\section{FRANCO ET LA CURE CHIRURGICALE DES HERNIES SANS ABLATION DU TESTICULE}

Franco rejeta, comme une pratique inutilement mutilante, la castration considérée comme nécessaire par la plupart des herniotomistes ambulants du Moyen Age. II montra que l'on pouvait très bien opérer les hernies, sans recourir à la suppression du testicule, en ménageant le cordon spermatique [12].

Franco, en pratiquant couramment la cure radicale des hernies, conservait le testicule ; quand le sac était trop adhérent aux organes voisins, il le laissait en place, mais fermait le péritoine par des sutures ; il liait et coupait l'épiploon après l'avoir cautérisé.

II faut mettre à l'actif de Franco une technique, qu'on trouve déjà dans son édition de 1556 , et dont il revendique expressément l'invention en la décrivant de nouveau en détail dans son édition de 1561, en intitulant ce chapitre, $D e$ cure de hernie par voie de chirurgie autrement, sans oster le testicule, de l'invention de l'autheur. Elle consiste à opérer à l'origine haute de la hernie, à isoler en fait le collet du sac, séparé avec précaution des vaisseaux spermatiques, tandis que le testicule maintenu par la traction d'un aide est attiré vers le bas ; et, après avoir appliqué la tenaille habituelle, à lier avec la précision qu'il décrit, en chaîne semble$t$-il, ce collet du sac et le couper. Franco abandonne donc le sac dans le scrotum ; mais, craignant le danger de rétention dans ce sac, il recommande de l'ouvrir à la partie intérieure du scrotum pour que la sanie puisse s'évacuer. Franco conseille cette opération tout au moins chez les monorchides ou en cas d'intervention bilatérale, car, étant plus complexe que l'intervention ordinaire, elle est à son avis hors de la compétence d'un opérateur non qualifié [11].

\section{DISCUSSION}

La lecture des œuvres de Franco permet de juger de sa valeur :

1. Comme homme: C'est un homme généreux, ayant le sens de l'humain, ne s'estimant satisfait qu'après avoir fait profiter d'autres chirurgiens de son expérience et de ses conseils.

2. Comme chirurgien : A la lecture des descriptions qu'il donne des signes observés par lui et des opérations qu'il préconise se dégage nettement l'impression qu'il avait beaucoup et bien travaillé, qu'il était instruit. Mais il n'était pas fait pour se contenter de copier ses prédécesseurs et de rapporter leurs idées. II n'écrit, en général, que ce qu'il a vu et fait.

3. Comme opérateur : II était adroit. II décrit minutieusement la technique qu'il recommande et, ainsi qu'il l'a promis, livre toutes les remarques pertinentes qu'il sut faire sur les détails de technique, les fautes qu'il ne faut pas commettre, les dangers à éviter, les correctifs qu'il conseille. On voit se dérouler une opération méthodiquement appuyée sur une grande rigueur anatomique, assurée par des gestes précis, prudents, dont aucun n'est laissé au hasard ni ne semble inutile.

4. Comme novateur: Son œuvre tire toute sa valeur de son invention personnelle. Sa part dans les progrès de la chirurgie est considérable : soit qu'il ait précisé certaines indications opératoires et perfectionné la technique pour des cas déjà connus, soit qu'il ait su distinguer des variétés nouvelles inaperçues et jusqu'alors confondues dans les descriptions classiques, soit qu'il ait imaginé et réalisé des méthodes opératoires originales permettant des actions plus osées en autorisant des indications plus larges en utilisant au besoin des instruments nouveaux de son invention [13].

Bien que Franco se soit élevé des rangs des chirurgiens incisives, il était inflexible en ses tentatives de retirer les traitements chirurgicales du domaine des charlatans itinérants et des coupeurs et de les placer sous les auspices de la médecine officielle. II a voulu élever la chirurgie opératoire à la position d'une partie respectée de la profession médicale.

\section{REFERENCES}

1. ANDROUTSOS G. : La taille vésicale et le serment hippocratique. Progrès en Urologie, 1995, $5:$ 426-440.

2. ARNAUD M., JOUVE P. : Pierre Franco, chirurgien provençal du $16^{\text {e }}$ siècle. Annales de Haute-Provence, 1958, 35, 215 : 113-128.

3. DIEULAFÉ R. : Progrès apportés par Pierre Franco à la chirurgie des hernies. Société Française d'Histoire de la Médecine, 1951, $4: 71-74$.

4. ELLIS H. : A History of Surgery, London, G.M.M., 2001 : 44.

5. FRANCO P. : Petit traité comprenant une des principales parties de chirurgie laquelle les chirurgiens herniaires exercent ainsi qu'il est montré en la page suivante. Lyon, Antoine Vincent, 1556. 
6. FRANCO P. : Traité des hernies et autres excellentes parties de la chirurgie assavoir la pierre. Lyon, Thibaud Payan, 1561.

7. HAEGER K. : The illustrated History of Surgery. London, Harold Starke, $1989: 147-148$.

8. HUARD P., GRMEK M. : La chirurgie moderne. Ses débuts en occident. Paris, Dacosta, 1968 : 99-107.

9. KÜSS G., HOUDARD L. : Pierre Franco. Traité des hernies. In: Commentaires sur dix grands livres de la médecine française. Paris, Claude Tchou, 1968 : 17-31.

10. LECENE P. : L'évolution de la chirurgie. Paris, Flammarion, $1923: 76$.

11. LIEUTAUD V. : Le célèbre Pierre Franco. Sisteron, A. Clergue, 1902.

12. NICAISE E. : Chirurgie de Pierre Franco. Paris, Alcan, 1895.

13. RUTKOW I. : Surgery. An Illustrated History. St Louis, Missouri, Mosby-Year Book, Inc., 1993 : 167-168.

14. STOPPA R., WANTZ G.E., MUNEGATO G., PUCHINOTTAA: Hernia Healers. An Illustated History. Velizy Villacoublay, Arnette, $1998: 20$.

15. VELTER A., LAMOTHE M.-J. : Les outils du corps. Paris, Cercle d'Art, 1984 : 154-155.

Manuscrit reçu : février 2004 ; accepté : février 2004.

\section{ABSTRACT}

Pierre Franco (1505-1578), an andrologist ahead of his time, who proposed testis-sparing hernia repair

\section{Georges ANDROUTSOS}

Pierre Franco (1505-1578), inventor of suprapubic lithotomy, cataract operation and testis-sparing hernia repair, is considered to be one of the most outstanding surgeons of the Renaissance and a great andrologist ahead of his time.

Key words: Pierre Franco, hernia repair, suprapubic lithotomy 